Revue de droit comparé du travail et de la sécurité sociale

2| 2021

La créativité du juge à l'épreuve des nouveaux problèmes de santé au travail

\title{
Reprise progressive des travaux à l'OIT
}

\section{Baptiste Delmas}

\section{(2) OpenEdition}

1 Journals

Édition électronique

URL : https://journals.openedition.org/rdctss/577

DOI : $10.4000 /$ rdctss. 577

ISSN : 2262-9815

Éditeur

Centre de droit comparé du travail et de la sécurité sociale

Édition imprimée

Date de publication : 1 juin 2021

Pagination : 110-115

ISSN : 2117-4350

\section{Référence électronique}

Baptiste Delmas, «Reprise progressive des travaux à I'OIT », Revue de droit comparé du travail et de la sécurité sociale [En ligne], 2 | 2021, mis en ligne le 01 juin 2021, consulté le 02 juin 2022. URL : http:// journals.openedition.org/rdctss/577 ; DOI : https://doi.org/10.4000/rdctss.577

\section{(c)}

La Revue de droit comparé du travail et de la sécurité sociale est mise à disposition selon les termes de la Licence Creative Commons Attribution - Pas d'Utilisation Commerciale - Pas de Modification 4.0 International. 


\section{BAPTISTE Delmas}

Post-doctorant en droit social, Comptrasec, UMR CNRS 5114, Université de Bordeaux

\section{REPRISE PROGRESSIVE DES TRAVAUX À L'OIT}

Après une mise sous cloche totale de ses activités institutionnelles en 2020 en raison de la pandémie de Covid-19, l'Organisation internationale du travail (OIT) reprend progressivement ses travaux.

Les $338^{e}$ et $339^{e}$ sessions du Conseil d'administration, qui devaient se tenir respectivement en mars et en juin 2020, avaient été annulées et la 109e session de la Conférence internationale du travail, initialement prévue en juin 2020, a quant à elle été reportée au mois de juin 2021. Elle se déroulera principalement sous forme virtuelle via une plateforme de visio-conférence en ligne ${ }^{1}$.

Mais la suspension des réunions qui rythment habituellement la vie de l'OIT n'a pas, pour autant, mis un coup de frein au fonctionnement interne de l'organisation.

C'est même, semble-t-il, le contraire. Aux ordres du jour récurrents s'ajoute, en effet, la gestion de l'incidence de la pandémie sur l'application et le respect des normes internationales du travail. C'est dire si l'actualité normative et institutionnelle de l'OIT est riche en ce printemps 2021.

Si le traitement des implications du SARS-COV-2 sur le marché du travail irrigue quasiment l'ensemble des documents publiés par I'OIT ces derniers mois, il faut sûrement en retenir au moins un, qui résume la position de l'Organisation sur le sujet. II s'agit de l'addendum au rapport de 2020 de la Commission d'experts pour l'application des conventions et recommandations soumis à la prochaine Conférence internationale ${ }^{2}$. Le rapport additionnel reprend à son compte le constat dramatique dressé en septembre 2020 par l'Observatoire de l'OIT sur le [sic] COVID-19³.

D'après cet addendum, les décisions de fermeture des lieux de travail prises par les gouvernements pour endiguer la pandémie auraient entraîné la perte de 495 millions d'emplois équivalents temps plein au deuxième trimestre de l'année 2020, 345 millions au troisième trimestre et 245 millions au dernier trimestre de la même année, soit un total d'un peu plus d'un milliard d'emplois ${ }^{4}$.

1 https://www.ilo.org/ilc/lLCSessions/109/lang--fr/index.htm

2 https://www.ilo.org/gb/GBSessions/GB341/ins/WCMS 771229/lang--fr/index.htm

3 https://www.ilo.org/global/topics/coronavirus/impacts-and-responses/WCMS 755930/lang-fr/index.htm.

4 Ibid., p. 1. 
Dans ce contexte, la Commission d'experts rappelle, en s'appuyant sur les observations publiées par d'autres organes onusiens ${ }^{5}$, que le respect des conventions déjà ratifiées continue, malgré tout, de s'imposer aux États. Plus particulièrement, les experts attirent la vigilance des mandants de I'OIT sur « l'accumulation exponentielle du pouvoir exécutif dans tous les pays ${ }^{6}$ pour faire face à la crise.

Les limitations apportées aux libertés et droits fondamentaux pour sauvegarder la santé publique, objectif légitime, doivent rester conformes aux " principes » de droit international, dont ceux de la légalité, de la nécessité, de la proportionnalité et de la non-discrimination. Enfin, la Commission d'experts constate que la pandémie mondiale témoigne de l'importance des normes internationales du travail en matière de santé et de sécurité.

Ce dernier rappel est loin d'être innocent. On se souvient que la Déclaration de I'OIT relative aux principes et droits fondamentaux au travail, adoptée en 1998, avait fait couler beaucoup d'encre, notamment en ce que le droit à un environnement de travail salubre n'y apparaissait pas. Le sujet était revenu au-devant de la scène lors des travaux précédant l'adoption de la Déclaration du Centenaire de l'OIT pour l'avenir du travail, adoptée en $2019^{7}$.

La proposition d'intégrer dans cette Déclaration une formule par laquelle " [I]a sécurité et la santé au travail constituent un principe et un droit fondamentaux au travail qui s'ajoutent à ceux énoncés dans la Déclaration de l'OIT relative aux principes et droits fondamentaux au travail, 1998 »'avait pas été retenue, l'affirmation plus neutre selon laquelle « des conditions de travail sûres et salubres sont fondamentales au travail décent " ayant été préférée (article II. D. de la Déclaration). Mais le compromis alors trouvé avait été de confier au Conseil d'administration le mandat « d'examiner, dans les meilleurs délais, des propositions visant à inclure la question des conditions de travail sûres et salubres dans le cadre de l'OIT relatif aux principes et droits fondamentaux au travail $»^{8}$.

C'est dans ce contexte qu'une première feuille de route a été soumise au Conseil d'administration, en octobre $2020^{\circ}$. Les départements du Portefeuille des politiques, ainsi que du Portefeuille des programmes extérieurs et des partenariats, à l'origine du document, envisagent les pistes possibles pour faire du droit à des conditions de travail sûres et salubres un droit fondamental.

Selon eux, il existerait deux méthodes possibles : la première serait d'amender la Déclaration de 1998, la seconde consisterait à adopter une Déclaration distincte,

5 Comité des droits économiques, sociaux et culturels, Déclaration sur la pandémie de maladie à coronavirus (COVID-19) et les droits économiques, sociaux et culturels, 17 avril 2020, doc. $n^{\circ} E / C .12 / 2020 / 1$; Comité des droits de l'Homme, Observation générale n³7 sur le droit de réunion pacifique, 17 septembre 2020, doc. $n^{\circ} \mathrm{CCPR} / \mathrm{C} / \mathrm{GC} / 37$. Ce renvoi confirme la volonté de I'OIT d'ancrer ses activités dans un paysage multilatéraliste. Voir A. Charbonneau, "L'avenir du travail en suspens », RDCTSS, 2020/2, p. 124.

6 Préc., note $1, n^{\circ} 51$.

7 I. Daugareilh, "La Déclaration du centenaire de I'OIT : tout un programme ! ", Droit social, 2020, n¹, p. 5.

8 Résolution sur la Déclaration du centenaire de l'OIT pour l'avenir du travail (adoptée le 21 juin 2019), Conférence internationale du Travail, 108 ession, 2019, n¹.

9 https://www.ilo.org/gb/GBSessions/GB340/ins/WCMS 757108/lang--fr/index.htm. 
propre à la question de la santé et de la sécurité au travail ${ }^{10}$. Mais les auteurs ne cachent pas leur préférence pour la première méthode, qui semble en effet être la plus heureuse. D'une part, parce qu'elle établirait un lien étroit entre la question des conditions de travail sûres et salubres et les principes et droits fondamentaux au travail existants. D'autre part, parce que, à l'inverse, l'adoption d'une énième Déclaration prendrait le risque de dilater encore un peu plus le tissu normatif de l'OIT et affaiblirait l'autorité de la reconnaissance du caractère fondamental d'un droit à des conditions de travail sûres.

Quatre instruments sont identifiés pour servir de soubassement constitutionnel à un tel droit fondamental :

- la Convention n¹55 sur la sécurité et la santé des travailleurs de 1981 ;

- la Convention n¹61 sur les services de santé au travail de 1985 ;

- la Convention $n^{\circ} 187$ sur le cadre promotionnel pour la sécurité et la santé au travail de 2006 ;

- le Protocole de 2002 relatif à la Convention $n^{0} 155^{11}$.

Enfin, le document n'ignore pas les difficultés que pourrait provoquer une telle consécration. Parmi elles, quid de la référence faite de plus en plus souvent par les accords de libre-échange et par les systèmes de préférence généralisée à la Déclaration de 1998 ? Il faut comprendre que ces accords étant de nature contractuelle, ce seront les parties qui décideront, librement, d'y ajouter ou non le droit fondamental à des conditions de travail sûres.

Ici, deux commentaires peuvent être formulés. D'abord, on y voit un argument supplémentaire à une modification de la Déclaration de 1998. Car l'adoption d'une Déclaration distincte pourrait inciter les négociateurs de futurs accords à procéder à un choix entre, d'un côté, la Déclaration de 1998 et, de l'autre, une Déclaration sur la santé et la sécurité. La référence à une Déclaration de 1998 modifiée emporterait l'obligation de respecter, en bloc, l'ensemble des droits fondamentaux au travail, sans démembrement possible.

Ensuite, le risque d'un law shopping pour les accords en vigueur peut être relativisé tant les effets accordés à une telle référence sont parfois limités. Par exemple, les accords de libre-échange conclus par l'Union européenne s'en tiennent le plus souvent à une vision incantatoire du respect des droits fondamentaux au travail.

À l'inverse, les modèles canadien et nord-américain d'accord de libre-échange soumettent le respect des droits fondamentaux au travail à la procédure d'arbitrage qu'ils instituent. Ce sont donc surtout pour ceux-là que les limites d'une modification de la Déclaration de 1998 se feraient sentir. Ces réflexions de fond devraient être abordées par le Conseil d'administration lors de sa $343^{\mathrm{e}}$ session, qui se tiendra normalement en novembre 2021, pour une éventuelle décision finale en juin 2022 à l'occasion de la $110^{\mathrm{e}}$ session de la Conférence internationale du travail ${ }^{12}$.

10 Ibid., $n^{\circ} 17$.

11 Ibid., n²7.

12 Décision concernant la suite à donner à la Résolution sur la Déclaration du centenaire de I'OIT pour l'avenir du travail: propositions visant à inclure la question des conditions de travail sûres et salubres dans le cadre des principes et droits fondamentaux au travail de I'OIT, 23 mars 2021, GB.341/INS/6/Décision. 
Si l'on reste sur le terrain de la santé et de la sécurité au travail, il faut signaler la ratification par la France, le 26 janvier 2021, de la Convention n¹84 sur la sécurité et la santé dans l'agriculture adoptée en 2001. Celle-ci entrera en vigueur le 26 janvier 2022. La France est le $19^{e}$ Etat membre à ratifier cette convention, le $11^{\mathrm{e}}$ sur le continent européen ${ }^{13}$. Cette ratification avait été annoncée par un projet de loi enregistré à la présidence du Sénat le 28 juin $2017^{14}$, et par la loi n²019-283 du 8 avril 2019 ${ }^{15}$. Elle n'est donc pas une surprise.

La Convention n¹84 de l'OIT poursuit l'objectif de prévenir la survenance d'accidents du travail et de maladies professionnelles dans le secteur agricole. Son champ d'application est particulièrement large puisque le terme " agriculture " est amplement défini à l'article $1^{\text {er }}$, et que les bénéficiaires escomptés de cette législation ne sont pas seulement les salariés mais aussi les indépendants, en particulier les chefs d'exploitation. Sont tour à tour abordés des risques de différente nature : utilisation des machines et ergonomie, manipulation et transport d'objets, contact avec des produits chimiques $^{16}$.

Enfin, la Convention réserve le cas particulier de publics jugés particulièrement vulnérables - jeunes travailleurs, travailleurs temporaires, travailleuses - et oblige les États signataires à adopter une règlementation portant sur le logement, l'aménagement du temps de travail et la couverture des accidents du travail et des maladies professionnelles.

Deux rapports, I'un rédigé par le Sénateur Joël Guerriau ${ }^{17}$ et l'autre par la Députée Martine Leguille-Balloy ${ }^{18}$ au moment de l'adoption de la loi autorisant la ratification, convergeaient pour dire que le droit français est déjà a priori en conformité avec le texte de la Convention. Un seul point s'était avéré, en réalité, litigieux.

Au moment de son adoption par I'OIT en 2001, la Fédération nationale des syndicats d'exploitants agricoles (FNSEA) avait émis des réserves quant à la ratification du texte en raison de son article 6. Celui-ci dispose que « la législation nationale ou l'autorité compétente devra prévoir que, sur un lieu de travail agricole, lorsque deux ou plus de deux employeurs exercent des activités ou lorsqu'un ou plusieurs employeurs et un ou plusieurs travailleurs indépendants exercent des activités, ils devront coopérer pour appliquer les prescriptions de sécurité et de santé ».

Or, si cette obligation de coopération existait déjà en droit français, c'était uniquement à l'égard des salariés et non pas des indépendants, pourtant bien visés par le texte international.

13 Après la Belgique, la Bosnie-Herzégovine, la Finlande, le Luxembourg, le Portugal, la République de Moldova, la Serbie, la Slovaquie, la Suède et I'Ukraine.

14 http://www.senat.fr/leg/pjl16-597.html

15 Loi n²019-283 du 8 avril 2019 autorisant la ratification de la Convention n¹84 de I'Organisation internationale du travail relative à la sécurité et la santé dans l'agriculture, JORF $\mathrm{n}^{\circ} 0084$ du 9 avril 2019.

16 On remarque que les risques liés au contact d'agents pathogènes zoonotiques ne sont pas abordés, si ce n'est indirectement à l'article 14 de la Convention qui porte sur la manipulation d'agents biologiques. La crise actuelle jette une lumière crue sur cette absence.

17 http://www.senat.fr/rap/l17-415//17-415.html

18 https://www.assemblee-nationale.fr/dyn/15/rapports/cion afetr//15b1364 rapport-fond\# 
En 2014, le législateur est donc intervenu et a inséré à l'article L. 717-10 du Code rural et de la pêche maritime une disposition selon laquelle « Les employeurs et travailleurs indépendants qui exercent les activités mentionnées aux $1^{\circ}, 2^{\circ}$ et $4^{\circ}$ de l'article L. 722-1 sur un même lieu de travail coopèrent afin de prévenir les risques résultant de leurs interventions simultanées ou successives et adoptent des mesures de prévention des risques professionnels appropriées. Les donneurs d'ordre concourent à la mise en œuvre de ces mesures ${ }^{19}$. L'exclusion expresse du troisième alinéa de l'article L. 722-1 du même Code, qui vise les travaux forestiers, s'explique par la présence d'une obligation analogue aux articles L. 717-8 et L. 717-9 du Code rural et de la pêche maritime ${ }^{20}$.

Si le droit français semble très proche des exigences de la Convention nouvellement ratifiée, il en va différemment de la Recommandation $n^{\circ} 198$ sur la relation de travail de 2006. C'est ce qui ressort de l'étude d'ensemble qui lui est en partie consacrée pour la première fois et qui sera soumise à la prochaine Conférence internationale ${ }^{21}$.

La lecture de ce document s'avère passionnante pour qui s'intéresse aux formes atypiques d'emploi et à la gig economy. En effet, elle fournit de très nombreux éléments de droit comparé sur la qualification de la relation de travail salarié en même temps que des suggestions précises à l'attention des pouvoirs publics pour s'orienter dans la " révolution » numérique à l'œuvre.

Or, le moins que l'on puisse dire est que les choix jusqu'alors opérés par la France ne convergent pas vraiment avec ces orientations. Par exemple, l'étude invite les Etats à « supprimer toute incitation à déguiser ou à dissimuler la relation de travail $»^{22}$, que ce soit en encadrant strictement le recours aux contrats civils ou commerciaux, ou en établissant une présomption simple de salariat ${ }^{23}$.

La création du statut d'auto-entrepreneur en $2008^{24}$, donc après l'adoption de la Recommandation $n^{\circ} 198$, et la lettre de mission adressée à Jean-Yves Frouin à l'origine du rapport éponyme ${ }^{25}$, ne vont pas vraiment dans cette direction. De même, l'étude suggère la mise en place d'un mécanisme périodique chargé " de proposer des mesures visant à ajuster les dispositions juridiques pertinentes ou leur application $»^{26}$ dans lequel devraient siéger les organisations de travailleurs et d'employeurs les plus représentatives. Là encore, ce n'est pas l'option retenue par le gouvernement français

19 Loi n²014-1170 du 13 octobre 2014 d'avenir pour l'agriculture, l'alimentation et la forêt, JORF n0238 du 14 octobre 2014, article 19.

20 Lesquels renvoient aux articles R. 717-78 et suivants du même Code.

21 https://www.ilo.org/ilc/ILCSessions/109/reports/reports-to-the-conference/WCMS 738280/ lang--fr/index.htm et son addendum suite à la crise sanitaire : https://www.ilo.org/ilc/ ILCSessions/109/reports/reports-to-the-conference/WCMS 775963/lang--fr/index.htm

$22 \S 194$.

23 Ibid., $\$ 233$.

24 Loi n²008-776 du 4 août 2008 de modernisation de l'économie, JORF n0181 du 5 août 2008.

25 https://www.gouvernement.fr/sites/default/files/document/document/2020/12/rapport reguler les plateformes numeriques de travail.pdf

26 Ibid., n³32. 
qui a préféré confier à Bruno Mettling, ancien DRH du groupe Orange, le soin de "proposer une architecture des mesures législatives » à la suite du rapport Frouin ${ }^{27}$.

Les échanges que suscitera cette étude lors de la Conférence internationale du mois de juin 2021 méritent donc sûrement que l'on s'y intéresse de près.

27 La lettre de mission délivrée par le Ministère du travail est accessible à : https://leplusimportant. org/documents/ 2021/03/lettre-de-mission-task-force-travailleurs-plateformes.pdf/ 